\title{
The outcome of intravenous and inhalation anesthesia after pancreatic cancer resection: a retrospective study
}

\author{
Yu Wang ( $D$ 86379317@qq.com ) \\ Harbin Medical University Cancer Hospital \\ Jie Ren \\ Harbin Medical University Cancer Hospital \\ Junli Wang \\ Harbin Medical University Cancer Hospital \\ Jingwen Chen \\ Harbin Medical University Cancer Hospital \\ Yue Ma \\ Harbin Medical University Cancer Hospital \\ Yutong Yang \\ Harbin Medical University Cancer Hospital \\ Ming Wei \\ Harbin Medical University Cancer Hospital \\ Liping Wang \\ Harbin Medical University Cancer Hospital
}

\section{Research Article}

Keywords: TIVA, INHA, SIPTW, Pancreatic cancer, Overall survival, Disease-free survival

Posted Date: February 24th, 2022

DOI: https://doi.org/10.21203/rs.3.rs-1262656/v1

License: (a) (i) This work is licensed under a Creative Commons Attribution 4.0 International License. Read Full License 


\section{Abstract}

Background: Different types of anesthesia may affect cancer patient's outcomes, we compared the overall survival (OS) and disease-free survival (DFS) of patients with pancreatic cancer under total intravenous and inhalation anesthesia.

Methods: The authors collected the electronic medical records of patients who had accepted at a pancreatectomy from January 1, 2010 to December 31, 2016. Patients respectively received total intravenous anesthesia (TIVA) or inhalational anesthesia (INHA). Stabilized inverse probability of treatment weighting (SIPTW)was used to minimize differences. Kaplan-Meier survival was established to analyze the influence of sort of anesthesia on disease-free and overall survival. We compare the effects of each sort of anesthesia on cancer recurrence or metastasis and all-cause mortality.

Results: A total of 463 patients who had undergone pancreatic cancer resection were collected in this study, of which 442 patients were available (TIVA group, $n=323$, INHA group, $n=119$ ). After SIPTW there were no significant differences between the two groups in disease-free survival (hazard ratio, $1.00,95 \% \mathrm{Cl}, 0.79$ to 1.27 , $P=0.995$ ) or overall survival (hazard ratio, $1.10,95 \% \mathrm{Cl}, 0.86$ to $1.41, P=0.433$ ).

Conclusions: In conclusion, the present study showed no significant difference in overall survival and diseasefree survival between total intravenous anesthesia and volatile anesthesia.

\section{Background}

Pancreatic cancer (PC) is one of the most common gastrointestinal malignancies with a five-year survival rate of only $10 \% .{ }^{[1]}$ Surgery is a common treatment for pancreatic cancer, which is diagnosed late due to a lack of effective screening methods. ${ }^{[2]}$ Although the tumor is removed by surgery, it also inevitably enters the blood circulation or lymphatic circulation and migrates to distant organs, causing tumor recurrence and metastasis.

${ }^{[3]}$ In addition, surgical trauma and the use of anesthetic drugs in the perioperative period can inhibit the body's anti-tumor immunity. ${ }^{[4]}$ The effect of different anesthetic drugs on tumor cells and tumor patients has always been a research hotspot. ${ }^{[5,6]}$

Studies have shown that propofol can exert anti-tumor effects through various mechanisms, including inhibiting tumor viability, inhibiting tumor progression, inhibiting cancer cell invasion, etc. ${ }^{[7,8]}$ In contrast, sevoflurane exhibited immunosuppression and tumorigenesis through a number of mechanisms, including suppression of natural killer (NK) cell activity and lymphocyte function, which induce proliferation, apoptosis, and invasion of cancer cells. ${ }^{[9,10]}$

In the prior research, we have proved an association between total intravenous anesthesia (TIVA) and the improvement of overall survival for gastric cancer (GC)patients who underwent resection. ${ }^{[11]}$ Therefore, we continue the relevant retrospective study to compare the overall survival and disease-free survival of patients after pancreatic cancer surgery with propofol-based TIVA and sevoflurane-based INHA.

\section{Methods}




\section{Patient identification and exclusion}

Cases of PC were identified from the records of patients and patients admitted to the hospital for cases resection between 2010 and 2016. Patients with metastasis, emergency operations, laparoscopic procedures and incomplete clinical data were excluded. ${ }^{[11,13]}$ Patients who experienced anesthesia and postoperative pathologies with PC were included. Medical records for all of the included patients were obtained, and the data were extracted by researchers who were not involved in the study or data analysis.

\section{Anesthesia technique and grouping method}

In both groups, anesthesia was induced with midazolam 0.05-0.15 mg/kg, $0.5 \mu \mathrm{g} / \mathrm{kg}$ fentanyl, and 1-2.5 $\mathrm{mg} / \mathrm{kg}$ propofol. ${ }^{[11]}$ Patients were divided into TIVA and INHA groups according to different anesthesia techniques. In the TIVA group, anesthesia was maintained with propofol and remifentanil. In the INHA group, anesthesia was maintained with sevoflurane and remifentanil. The postoperative pain management methods were the same in the two groups and neither has undergone epidural anesthesia.

\section{Indicator and data}

The statuses of patients up to November 30, 2019 were determined from medical records and causes of death were record. We obtained the following information: demographic data, cancer stage, degree of differentiation, American Society of Anesthesiologists (ASA) grade, duration of surgery, primary diagnosis, transfusion, preoperative or postoperative adjuvant chemotherapy, and/or radiation therapy were received. ${ }^{[6,11]}$ Cancer stage was assessed based on the 8th edition of American Joint Committee on Cancer (AJCC) Cancer Staging Manual. ${ }^{[32]}$ The degrees of differentiation included well differentiated and poor differentiated. The primary diagnosis included pancreatic head cancer, pancreatic body or tail cancer. Types of surgery included distal pancreatectomy, pancreaticoduodenectomy and other kinds of pancreatectomy. Survival time was measured from the date of pancreatectomy to death or to the last followed-up before November 30, 2019.

The primary endpoint of this study was overall survival (OS), which was defined as the period from the patient's date of surgery to the time of death. The secondary endpoint was disease-free survival (DFS), which was defined as the interval between the date of surgery and the date of tumor recurrence and metastasis or death.

\section{Statistical approach}

The cases with unqualified data were excluded from the final analysis, we analyzed the cases that meet the requirements in this study. Fisher's exact text or $\chi 2$ test was used to evaluate the associations between categorical variables. T-tests or Manne Whitney $U$ tests were used to compare continuous variables between patient groups. Categorical data was expressed as $n(\%)$ and analysed with the $\chi 2$ test, continuous data was expressed as the mean (standard deviation, SD) or median [interquartile range], and two independent samples were analysed with the T-test. ${ }^{[6,12]}$ 
The Kaplan Meier method was used to calculate OS and DFS. Cox proportional hazards regression models were used to compare risk factors between the different groups by using univariate models. Significant variables in univariate analysis and clinically significant variables were entered into multivariate analysis. Propensity score matching was used to reduce the difference between groups, which would inevitably reduce the sample size. Thus, we chose stabilized inverse probability of treatment weighting (SIPTW) to make a good balance ${ }^{[13]}$. These variables were entered in our propensity model: age, sex, ASA physical status, duration of surgery, degree of differentiation, cancer stage, surgery type, hypertension, smoke, blood transfusion, diabetes, drink, tumor location and adjuvant treatment. All analyses were performed using $\mathrm{R}$ software version 4.1.2(R Foundation for Statistical Computing, Austria). We used the package "survival" for the Cox regression analysis and package "IPW survival" for the stabilized inverse probability of treatment weighting. Forest plot was built by "forestplot" package and p-value $<0.05$ was considered statistically significant.

\section{Results}

This retrospective analysis of 463 patients who underwent pancreatectomy for PC were enrolled in this study. After the inclusion criteria were applied, 119 patients were in the propofol intravenous group and 323 patients were in the inhalation group (Fig. 1). The SIPTW procedure was performed to adjust for imbalances in these retrospective settings. After stabilized inverse probability of treatment weighting, the sum of weights was 118.7 in the TIVA group, and 323.1 in the INHA group. All standardized mean differences (SMD)for the study variables were less than 0.1 (Table 1 ). 
Table 1

Patient Characteristics for before SIPTW adjustment and after SIPTW adjustment

\begin{tabular}{|c|c|c|c|c|c|c|c|c|}
\hline \multicolumn{2}{|l|}{ Varible } & \multicolumn{3}{|c|}{$\begin{array}{l}\text { Before SIPTW } \\
\text { adjustment }\end{array}$} & \multicolumn{4}{|c|}{$\begin{array}{l}\text { After SIPTW } \\
\text { adjustment }^{\text {a }}\end{array}$} \\
\hline & & INHA & TIVA & $P$ & INHA & TIVA & $P$ & $S M D^{b}$ \\
\hline & & $\begin{array}{l}n= \\
323\end{array}$ & $\mathrm{n}=119$ & & $\begin{array}{l}\text { Sum } \\
\text { of } \\
\text { weight }\end{array}$ & $\begin{array}{l}\text { Sum } \\
\text { of } \\
\text { weight }\end{array}$ & & \\
\hline & & & & & $\begin{array}{l}= \\
323.1\end{array}$ & $=$ & & \\
\hline \multirow[t]{2}{*}{ Sex (\%) } & Female & $\begin{array}{l}139 \\
(43.0)\end{array}$ & $\begin{array}{l}53 \\
(44.5)\end{array}$ & 0.861 & $\begin{array}{l}141.1 \\
(43.7)\end{array}$ & $\begin{array}{l}51.8 \\
(43.6)\end{array}$ & 0.995 & 0.001 \\
\hline & Male & $\begin{array}{l}184 \\
(57.0)\end{array}$ & $\begin{array}{l}66 \\
(55.5)\end{array}$ & & $\begin{array}{l}182.0 \\
(56.3)\end{array}$ & $\begin{array}{l}66.9 \\
(56.4)\end{array}$ & & \\
\hline $\begin{array}{l}\text { Age (median-IQR, } \\
\text { year) }\end{array}$ & & $\begin{array}{l}57.00 \\
{[51.00} \\
63.00]\end{array}$ & $\begin{array}{l}57.00 \\
{[50.00,} \\
62.00]\end{array}$ & 0.544 & $\begin{array}{l}57.00 \\
{[51.00} \\
62.09]\end{array}$ & $\begin{array}{l}57.00 \\
{[50.38,} \\
62.00]\end{array}$ & 0.749 & 0.012 \\
\hline $\begin{array}{l}\text { BMI (median- } \\
\text { IQR,kg/m2) }\end{array}$ & & $\begin{array}{l}23.44 \\
{[21.41,} \\
25.81]\end{array}$ & $\begin{array}{l}22.93 \\
{[21.44,} \\
25.11]\end{array}$ & 0.289 & $\begin{array}{l}23.35 \\
{[21.27} \\
25.64]\end{array}$ & $\begin{array}{l}23.04 \\
{[21.63,} \\
25.44]\end{array}$ & 0.89 & 0.065 \\
\hline \multirow[t]{2}{*}{ Smoke (\%) } & No & $\begin{array}{l}180 \\
(55.7)\end{array}$ & $\begin{array}{l}70 \\
(58.8)\end{array}$ & 0.635 & $\begin{array}{l}183.7 \\
(56.9)\end{array}$ & $\begin{array}{l}67.6 \\
(57.0)\end{array}$ & 0.986 & 0.002 \\
\hline & Yes & $\begin{array}{l}143 \\
(44.3)\end{array}$ & $\begin{array}{l}49 \\
(41.2)\end{array}$ & & $\begin{array}{l}139.4 \\
(43.1)\end{array}$ & $\begin{array}{l}51.1 \\
(43.0)\end{array}$ & & \\
\hline \multirow[t]{2}{*}{ Drink (\%) } & No & $\begin{array}{l}246 \\
(76.2)\end{array}$ & $\begin{array}{l}88 \\
(73.9)\end{array}$ & 0.722 & $\begin{array}{l}245.3 \\
(75.9)\end{array}$ & $\begin{array}{l}91.3 \\
(76.9)\end{array}$ & 0.827 & 0.023 \\
\hline & Yes & $\begin{array}{l}77 \\
(23.8)\end{array}$ & $\begin{array}{l}31 \\
(26.1)\end{array}$ & & $\begin{array}{l}77.7 \\
(24.1)\end{array}$ & $\begin{array}{l}27.4 \\
(23.1)\end{array}$ & & \\
\hline \multirow[t]{2}{*}{ Hypertension (\%) } & No & $\begin{array}{l}263 \\
(81.4)\end{array}$ & $\begin{array}{l}96 \\
(80.7)\end{array}$ & 0.966 & $\begin{array}{l}262.7 \\
(81.3)\end{array}$ & $\begin{array}{l}96.5 \\
(81.3)\end{array}$ & 0.995 & 0.001 \\
\hline & Yes & $\begin{array}{l}60 \\
(18.6)\end{array}$ & $\begin{array}{l}23 \\
(19.3)\end{array}$ & & $\begin{array}{l}60.4 \\
(18.7)\end{array}$ & $\begin{array}{l}22.2 \\
(18.7)\end{array}$ & & \\
\hline \multirow[t]{2}{*}{ Diabetes (\%) } & No & $\begin{array}{l}277 \\
(85.8)\end{array}$ & $\begin{array}{l}99 \\
(83.2)\end{array}$ & 0.603 & $\begin{array}{l}275.7 \\
(85.3)\end{array}$ & $\begin{array}{l}102.3 \\
(86.2)\end{array}$ & 0.809 & 0.025 \\
\hline & Yes & $\begin{array}{l}46 \\
(14.2)\end{array}$ & $\begin{array}{l}20 \\
(16.8)\end{array}$ & & $\begin{array}{l}47.4 \\
(14.7)\end{array}$ & $\begin{array}{l}16.4 \\
(13.8)\end{array}$ & & \\
\hline
\end{tabular}

Abbreviations: IQR, inter-quartile range; Cancer stages: stage I: T1, NO, M0/T2, N0, MO/T1, N1, M0; stage II: T3, N0, MO/T4a, N1, M0/T3, N1, M0/T2, N2, M0/T1, N3, M0; stage III: T2, N3, M0/T3, N2, M0/T3, N3, $\mathrm{MO} / \mathrm{T} 4 \mathrm{a}, \mathrm{N} 2, \mathrm{M0} / \mathrm{T} 4 \mathrm{a}, \mathrm{N} 3, \mathrm{MO} /$ any $\mathrm{T} 4 \mathrm{~b}$, any N, M0; stage IV: any T, any N, M1.ASA, American Society of Anesthesiologists; BMI, body mass index; INHA, inhalational anesthesia; TIVA, total intravenous anesthesia, SIPTW, stabilized inverse probability of treatment weighting; SMD, Standardized Mean Difference 


\begin{tabular}{|c|c|c|c|c|c|c|c|c|}
\hline \multirow{2}{*}{$\begin{array}{l}\text { Varible } \\
\begin{array}{l}\text { Adjuvant } \\
\text { treatment(\%) }\end{array}\end{array}$} & \multirow[b]{2}{*}{ No } & \multicolumn{3}{|c|}{$\begin{array}{l}\text { Before SIPTW } \\
\text { adjustment }\end{array}$} & \multicolumn{4}{|c|}{$\begin{array}{l}\text { After SIPTW } \\
\text { adjustment }^{\text {a }}\end{array}$} \\
\hline & & $\begin{array}{l}225 \\
(69.7)\end{array}$ & $\begin{array}{l}86 \\
(72.3)\end{array}$ & 0.678 & $\begin{array}{l}227.2 \\
(70.3)\end{array}$ & $\begin{array}{l}83.8 \\
(70.6)\end{array}$ & 0.954 & 0.006 \\
\hline & Yes & $\begin{array}{l}98 \\
(30.3)\end{array}$ & $\begin{array}{l}33 \\
(27.7)\end{array}$ & & $\begin{array}{l}95.8 \\
(29.7)\end{array}$ & $\begin{array}{l}34.9 \\
(29.4)\end{array}$ & & \\
\hline \multirow[t]{2}{*}{$\begin{array}{l}\text { Blood } \\
\text { transfusion (\%) }\end{array}$} & No & $\begin{array}{l}196 \\
(60.7)\end{array}$ & $\begin{array}{l}80 \\
(67.2)\end{array}$ & 0.250 & $\begin{array}{l}200.5 \\
(62.1)\end{array}$ & $\begin{array}{l}72.3 \\
(60.9)\end{array}$ & 0.835 & 0.023 \\
\hline & Yes & $\begin{array}{l}127 \\
(39.3)\end{array}$ & $\begin{array}{l}39 \\
(32.8)\end{array}$ & & $\begin{array}{l}122.5 \\
(37.9)\end{array}$ & $\begin{array}{l}46.4 \\
(39.1)\end{array}$ & & \\
\hline $\begin{array}{l}\text { Duration } \\
\text { (median-IQR,h) }\end{array}$ & & $\begin{array}{l}4.00 \\
{[3.50,} \\
4.50]\end{array}$ & $\begin{array}{l}4.00 \\
{[3.50} \\
4.50]\end{array}$ & 0.820 & $\begin{array}{l}4.00 \\
{[3.50} \\
4.50]\end{array}$ & $\begin{array}{l}4.00 \\
{[3.50,} \\
4.50]\end{array}$ & 0.534 & 0.021 \\
\hline \multirow[t]{3}{*}{ ASA (\%) } & 1 & $7(2.2)$ & $4(3.4)$ & 0.282 & $\begin{array}{l}8.0 \\
(2.5)\end{array}$ & $\begin{array}{l}2.8 \\
(2.4)\end{array}$ & 0.997 & 0.008 \\
\hline & II & $\begin{array}{l}310 \\
(96.0)\end{array}$ & $\begin{array}{l}110 \\
(92.4)\end{array}$ & & $\begin{array}{l}307.0 \\
(95.0)\end{array}$ & $\begin{array}{l}112.8 \\
(95.0)\end{array}$ & & \\
\hline & III & $6(1.9)$ & $5(4.2)$ & & $\begin{array}{l}8.1 \\
(2.5)\end{array}$ & $\begin{array}{l}3.1 \\
(2.6)\end{array}$ & & \\
\hline \multirow[t]{2}{*}{$\begin{array}{l}\text { Tumour location } \\
(\%)\end{array}$} & Head & $\begin{array}{l}228 \\
(70.6)\end{array}$ & $\begin{array}{l}80 \\
(67.2)\end{array}$ & 0.572 & $\begin{array}{l}224.9 \\
(69.6)\end{array}$ & $\begin{array}{l}82.4 \\
(69.4)\end{array}$ & 0.962 & 0.005 \\
\hline & Tail or body & $\begin{array}{l}95 \\
(29.4)\end{array}$ & $\begin{array}{l}39 \\
(32.8)\end{array}$ & & $\begin{array}{l}98.2 \\
(30.4)\end{array}$ & $\begin{array}{l}36.4 \\
(30.6)\end{array}$ & & \\
\hline \multirow[t]{4}{*}{ Cancer stage(\%) } & I & $\begin{array}{l}158 \\
(48.9)\end{array}$ & $\begin{array}{l}51 \\
(42.9)\end{array}$ & 0.553 & $\begin{array}{l}152.4 \\
(47.2)\end{array}$ & $\begin{array}{l}56.3 \\
(47.5)\end{array}$ & 0.996 & 0.028 \\
\hline & $\|$ & $\begin{array}{l}124 \\
(38.4)\end{array}$ & $\begin{array}{l}50 \\
(42.0)\end{array}$ & & $\begin{array}{l}127.1 \\
(39.4)\end{array}$ & $\begin{array}{l}46.1 \\
(38.8)\end{array}$ & & \\
\hline & III & $\begin{array}{l}25 \\
(7.7)\end{array}$ & $\begin{array}{l}13 \\
(10.9)\end{array}$ & & $\begin{array}{l}28.5 \\
(8.8)\end{array}$ & $\begin{array}{l}11.2 \\
(9.4)\end{array}$ & & \\
\hline & IV & $\begin{array}{l}16 \\
(5.0)\end{array}$ & $5(4.2)$ & & $\begin{array}{l}15.0 \\
(4.7)\end{array}$ & $\begin{array}{l}5.1 \\
(4.3)\end{array}$ & & \\
\hline \multirow[t]{2}{*}{ Surgery type (\%) } & $\begin{array}{l}\text { Distal } \\
\text { pancreatectomy }\end{array}$ & $\begin{array}{l}101 \\
(31.3)\end{array}$ & $\begin{array}{l}41 \\
(34.5)\end{array}$ & 0.451 & $\begin{array}{l}104.1 \\
(32.2)\end{array}$ & $\begin{array}{l}38.5 \\
(32.4)\end{array}$ & 0.999 & 0.004 \\
\hline & $\begin{array}{l}\text { Pancreatico- } \\
\text { duodenectomy }\end{array}$ & $\begin{array}{l}220 \\
(68.1)\end{array}$ & $\begin{array}{l}76 \\
(63.9)\end{array}$ & & $\begin{array}{l}216.1 \\
(66.9)\end{array}$ & $\begin{array}{l}79.2 \\
(66.7)\end{array}$ & & \\
\hline
\end{tabular}

Abbreviations: IQR, inter-quartile range; Cancer stages: stage I: T1, N0, MO/T2, NO, M0/T1, N1, M0; stage II: T3, NO, MO/T4a, N1, MO/T3, N1, M0/T2, N2, M0/T1, N3, M0; stage III: T2, N3, M0/T3, N2, M0/T3, N3, $\mathrm{MO} / \mathrm{T} 4 \mathrm{a}, \mathrm{N} 2, \mathrm{MO} / \mathrm{T} 4 \mathrm{a}, \mathrm{N} 3, \mathrm{MO}$ /any $\mathrm{T} 4 \mathrm{~b}$, any $\mathrm{N}$, M0; stage IV: any T, any N, M1.ASA, American Society of Anesthesiologists; BMI, body mass index; INHA, inhalational anesthesia; TIVA, total intravenous anesthesia, SIPTW, stabilized inverse probability of treatment weighting; SMD, Standardized Mean Difference 


\begin{tabular}{|c|c|c|c|c|c|c|c|c|}
\hline \multirow[t]{2}{*}{ Varible } & \multirow[b]{2}{*}{ Other } & \multicolumn{3}{|c|}{$\begin{array}{l}\text { Before SIPTW } \\
\text { adjustment }\end{array}$} & \multicolumn{4}{|c|}{$\begin{array}{l}\text { After SIPTW } \\
\text { adjustment }^{\text {a }}\end{array}$} \\
\hline & & $2(0.6)$ & $2(1.7)$ & & $\begin{array}{l}2.9 \\
(0.9)\end{array}$ & $\begin{array}{l}1.1 \\
(0.9)\end{array}$ & & \\
\hline \multirow[t]{2}{*}{$\begin{array}{l}\text { Degree of } \\
\text { differentiation(\%) }\end{array}$} & Poor & $\begin{array}{l}119 \\
(36.8)\end{array}$ & $\begin{array}{l}46 \\
(38.7)\end{array}$ & 0.811 & $\begin{array}{l}120.0 \\
(37.1)\end{array}$ & $\begin{array}{l}43.1 \\
(36.3)\end{array}$ & 0.88 & 0.016 \\
\hline & Well & $\begin{array}{l}204 \\
(63.2)\end{array}$ & $\begin{array}{l}73 \\
(61.3)\end{array}$ & & $\begin{array}{l}203.1 \\
(62.9)\end{array}$ & $\begin{array}{l}75.6 \\
(63.7)\end{array}$ & & \\
\hline \multicolumn{9}{|c|}{ 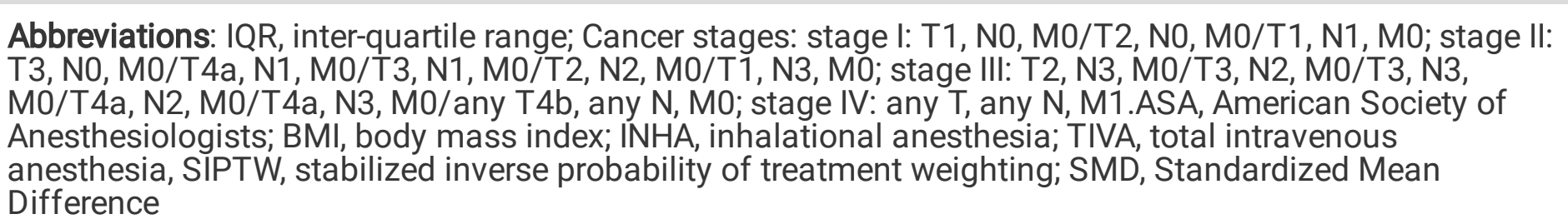 } \\
\hline
\end{tabular}

In this study, the median follow-up time for all patients was 18.5 months (interquartile range, 10 to 35.5 ). TIVA group was 17.5 months (interquartile range, 12 to 31.5 ) and INHA group was 18.5 months (interquartile range, 9.5 to 37.5). The Kaplan-Meier survival curves demonstrated the OS rates for 1-year and 3-year in TIVA were $73.5 \%(95 \% \mathrm{Cl}, 68.9-82 \%), 25.9 \%(95 \mathrm{Cl} \%, 18.5-36.4 \%)$ and in INHA $70.4 \%(95 \% \mathrm{Cl}, 65.5-75.7 \%), 32.6 \%$ (95CI\%, 27.6-38.5\%). The DFS rates for 1-year and 3-year in TIVA were 56.7\% (48.2-66.6\%), 23.1\% $(95 \% \mathrm{Cl}, 18.8-28.4 \%)$ and in INHA were $55.3 \%(95 \% \mathrm{Cl}, 50.1-61.1 \%), 21.0 \%(95 \% \mathrm{Cl}, 14.3-30.7 \%)$. There was no significant difference in overall survival ( $p$-value $=0.360$ ) or disease-free survival ( $p$-value $=0.681$ ) between the TIVA group and the INHA group in the SIPTW cohort (Fig. 2a,2b).

In the SIPTW cohort, the Cox proportional hazards model for overall survival and disease-free survival were built to evaluate the association between type of anesthesia and overall survival or disease-free survival. Univariate Cox regression revealed no significant association between TIVA and poorer OS (hazard ratio = $0.96,95 \% \mathrm{Cl}, 0.90$ to $1.43, p=0.298$ ) or DFS (hazard ratio $=1.05,95 \% \mathrm{Cl}, 0.84$ to $1.32, p=0.66$ ) when compared with the INHA group (Table 2). 
Table 2

Univariate analysis of OS and DFS.

\begin{tabular}{|c|c|c|c|c|c|}
\hline \multicolumn{2}{|l|}{ Varibles } & \multicolumn{2}{|l|}{ OS(SIPTW) } & \multicolumn{2}{|l|}{ DFS(SIPTW) } \\
\hline & & HR.Cl95 & $\begin{array}{l}p- \\
\text { value }\end{array}$ & HR.Cl95 & $\begin{array}{l}p- \\
\text { value }\end{array}$ \\
\hline Age & & $\begin{array}{l}1.02(1.01- \\
1.03)\end{array}$ & 0.001 & $\begin{array}{l}1.01(1.00- \\
1.02)\end{array}$ & 0.025 \\
\hline Sex & & $\begin{array}{l}0.96(0.77- \\
1.20)\end{array}$ & 0.728 & $\begin{array}{l}0.87(0.70- \\
1.07)\end{array}$ & 0.186 \\
\hline BMI & & $\begin{array}{l}0.96(0.92- \\
0.99)\end{array}$ & 0.034 & $\begin{array}{l}0.98(0.95- \\
1.01)\end{array}$ & 0.244 \\
\hline \multirow[t]{2}{*}{ Group } & INHA & & & & \\
\hline & TIVA & $\begin{array}{l}1.13(0.90- \\
1.43)\end{array}$ & 0.298 & $\begin{array}{l}1.05(0.84- \\
1.32)\end{array}$ & 0.660 \\
\hline \multirow[t]{2}{*}{ Smoke } & No & & & & \\
\hline & Yes & $\begin{array}{l}1.15(0.92- \\
1.45)\end{array}$ & 0.220 & $\begin{array}{l}0.91(0.73- \\
1.13)\end{array}$ & 0.397 \\
\hline \multirow[t]{2}{*}{ Drink } & No & & & & \\
\hline & Yes & $\begin{array}{l}1.03(0.79- \\
1.33)\end{array}$ & 0.850 & $\begin{array}{l}0.89(0.69- \\
1.15)\end{array}$ & 0.372 \\
\hline \multirow[t]{2}{*}{ Hypertension } & No & & & & \\
\hline & Yes & $\begin{array}{l}1.16(0.87- \\
1.55)\end{array}$ & 0.321 & $\begin{array}{l}0.98(0.75- \\
1.28)\end{array}$ & 0.884 \\
\hline \multirow[t]{2}{*}{ Diabetes } & No & & & & \\
\hline & Yes & $\begin{array}{l}0.86(0.59- \\
1.24)\end{array}$ & 0.424 & $\begin{array}{l}0.85(0.62- \\
1.19)\end{array}$ & 0.346 \\
\hline \multirow{2}{*}{$\begin{array}{l}\text { Adjuvant } \\
\text { treatment }\end{array}$} & No & & & & \\
\hline & Yes & $\begin{array}{l}0.65(0.51- \\
0.85)\end{array}$ & 0.001 & $\begin{array}{l}0.65(0.51- \\
0.83)\end{array}$ & $<0.001$ \\
\hline \multirow[t]{2}{*}{ Bloodtransfusion } & No & & & & \\
\hline & Yes & $\begin{array}{l}1.19(0.95- \\
1.50)\end{array}$ & 0.125 & $\begin{array}{l}1.26(1.01- \\
1.57)\end{array}$ & 0.038 \\
\hline Duration & Duration & $\begin{array}{l}1.15(1.05- \\
1.25)\end{array}$ & 0.003 & $\begin{array}{l}1.11(1.02- \\
1.20)\end{array}$ & 0.019 \\
\hline \multirow[t]{2}{*}{ ASA } & I & & & & \\
\hline & $\|$ & $\begin{array}{l}1.77(0.83- \\
3.77)\end{array}$ & 0.139 & $\begin{array}{l}2.19(1.05- \\
4.57)\end{array}$ & 0.037 \\
\hline
\end{tabular}




\begin{tabular}{|c|c|c|c|c|c|}
\hline \multirow[t]{2}{*}{ Varibles } & \multirow[b]{2}{*}{ III } & \multicolumn{2}{|l|}{ OS(SIPTW) } & \multicolumn{2}{|l|}{ DFS(SIPTW) } \\
\hline & & $\begin{array}{l}4.52(1.70- \\
12.00)\end{array}$ & 0.003 & $\begin{array}{l}6.99(2.99- \\
16.32)\end{array}$ & $<0.001$ \\
\hline \multirow[t]{2}{*}{ Tumour location } & Head & & & & \\
\hline & Tail or body & $\begin{array}{l}0.83(0.64- \\
1.07)\end{array}$ & 0.149 & $\begin{array}{l}1.01(0.79- \\
1.30)\end{array}$ & 0.931 \\
\hline \multirow[t]{4}{*}{ Cancer stage } & I & & & & \\
\hline & II & $\begin{array}{l}0.99(0.78- \\
1.26)\end{array}$ & 0.970 & $\begin{array}{l}1.20(0.96- \\
1.50)\end{array}$ & 0.110 \\
\hline & III & $\begin{array}{l}1.18(0.76- \\
1.83)\end{array}$ & 0.450 & $\begin{array}{l}1.57(0.99- \\
2.51)\end{array}$ & 0.060 \\
\hline & IV & $\begin{array}{l}1.39(0.75- \\
2.57)\end{array}$ & 0.290 & $\begin{array}{l}1.90(1.07- \\
3.38)\end{array}$ & 0.030 \\
\hline \multirow[t]{3}{*}{ Surgery type } & Distal pancreatectomy & & & & \\
\hline & Other & $\begin{array}{l}1.13(0.23- \\
5.63)\end{array}$ & 0.881 & $\begin{array}{l}0.74(1.18- \\
3.06)\end{array}$ & 0.673 \\
\hline & Pancreaticoduodenectomy & $\begin{array}{l}1.27(0.98- \\
1.63)\end{array}$ & 0.071 & $\begin{array}{l}1.06(0.83- \\
1.35)\end{array}$ & 0.628 \\
\hline \multirow{2}{*}{$\begin{array}{l}\text { Degree of } \\
\text { differentiation }\end{array}$} & Poor & & & & \\
\hline & Well & $\begin{array}{l}0.73(0.58- \\
0.92)\end{array}$ & 0.009 & $\begin{array}{l}0.70(0.56- \\
0.87)\end{array}$ & 0.002 \\
\hline
\end{tabular}

In the multivariate Cox model considered that include factors which are $p<0.05$ in the univariate Cox regression or clinically significant factors. The results were shown in the forest plots that there were no significant between TIVA and IHNA to improve OS (HR $=1.10,95 \% \mathrm{Cl}, 0.86$ to $1.41, p=0.433)$ or DFS (HR = $1.00,95 \% \mathrm{Cl}, 0.79$ to $1.27, p=0.995)$ in $\mathrm{PC}$ patients. Adjuvant treatment $(\mathrm{HR}=0.69,95 \% \mathrm{Cl}, 0.53$ to $0.91, p=0.008$, $\mathrm{HR}=0.69,95 \% \mathrm{Cl}, 0.53$ to $0.89, p=0.005)$ and Degree of differentiation ( $\mathrm{HR}=0.73,95 \% \mathrm{Cl}, 0.58$ to $0.94, p=0.013$, $\mathrm{HR}=0.69,95 \% \mathrm{Cl}, 0.55$ to $0.86, p=0.001)$ remained statistically significant in improving OS and DFS (Fig. 3a,3b).

\section{Discussion}

This study demonstrated no significant correlation between total intravenous anesthesia (TIVA) and better overall survival (OS) in patients who underwent PC surgery. We also found that total intravenous anesthesia did not improve the disease-free survival (DFS) of patients. The results of this study inconsistent with a study that propofol-based TIVA can enhance the prognosis of patients with PC ${ }^{[14]}$.

In recent years, the prognosis of anesthetics for various tumor patients was investigated. One study found that total intravenous anesthesia based on propofol had a better survival in colon cancer. ${ }^{[15]}$ Another study 
conducted a retrospective cohort study on gastric cancer surgeries in 2856 patients, and reported improved overall survival in the TIVA group, compared to the inhalation group ${ }^{[11]}$. In contrast, some retrospective studies proved no difference between the TIVA group and inhalation group for overall survival in digestive cancer surgery. ${ }^{[16,17]}$ The available data suggested that the impact of propofol based TIVA on long term outcome of cancer surgery is still controversial currently.

The immunomodulatory effect of anesthetics was considered to be the vital mechanism by which anesthesia affects the prognosis of cancer. Both in vivo and in vitro experiments had shown that inhalation anesthesia inhibits the toxicity of NK cells, which was critical to prevent tumor growth. ${ }^{[18,19]}$ Furthermore, several studies had shown that volatile anesthetic agents induce upregulation of tumorigenic growth factors, including hypoxia-inducible factor- 1 and serum inflammatory factor. ${ }^{[20,21]}$ Corresponding to propofol, it had been found to enhance the activity of NK cells, reduce tumour inflammatory factors, and have protective and anticancer effects. ${ }^{[22,23]}$ However, there were also some studies showing that the two anesthetics had little effect on immune cells and the inhibition and activation of the two types of anesthesia drugs may depend on clinical conditions. ${ }^{[20,24]}$

In our research, we have included more patients than previous study ${ }^{[14]}$, and adopted a new method to reduce the difference between the two groups. Retrospective studies ${ }^{[11,14]}$ usually used propensity score matching to reduce confounding between groups. Propensity score matching can cause the loss of a large amount of data. IPTW can increase the amount of data several times and increases the false positive rate. ${ }^{[13]}$ SIPTW not only balanced the confounding factors of the two groups of people, but also kept the amount of data indistinguishable from the original. ${ }^{[13]}$ DFS might replace OS as a surrogate endpoint for cancer patients, ${ }^{[25]}$ but it had not been proven in pancreatic cancer. ${ }^{[26]}$ OS is still the main criterion for judging the survival of PC patients. Therefore, our study took OS as the main target and DFS as a comparison and supplement. In the multivariate COX risk proportional regression model, OS was related to age. Adjuvant treatment and degree of differentiation were significantly correlated with OS, which was consistent with the results of previous studies $^{[12,27]}$. However, age and BMI had nothing to do with the length of DFS, which showed a significant correlation in ASAIII. ASA was often used to assess patient status, it has been proved that patient status was related to metastasis and recurrence ${ }^{[28]}$. But the amount of data was too small and the persuasive ability of the data was too weak. In addition, Cancer stage II and Cancer stage IV were related to DFS and there was also no correlation between the type of surgery and OS or DFS.

The effect of perioperative blood transfusion on tumor prognosis has always been controversial. In a retrospective study, perioperative blood transfusion was associated with OS and DFS in patients with stage I to III gastric cancer who underwent tumor resection, and the amount of transfusion OS and DFS showed a non-linear dose relationship. ${ }^{[29]}$ Another multicenter regression study found that perioperative blood transfusion among patients undergoing radical rectal cancer surgery was associated with worse OS, not with worse DFS. ${ }^{\left[{ }^{30}\right]}$ When restricted to pancreatic cancer, previous studies have found no relationship between blood transfusion and $\mathrm{OS}^{[14]}$ another review suggests that there is currently no conclusive evidence that PC is associated with perioperative blood transfusion. ${ }^{[31]}$ Our study verified that OS in pancreatic cancer was not correlated with blood transfusion, and DFS was also not correlated with blood transfusion. 
There were some inevitable limitations to our study. Firstly, we did not collect certain clinical data, such as specific medication, perioperative opioid use. While the impact of opioids on the prognosis of tumors was still controversial, and the literature showed that it did not affect the survival rate of PC. ${ }^{[27]}$ Secondly, the sample size was single, which required multi-center and larger clinical data. Thirdly, because of the retrospective study design, it was not possible to measure levels of inflammatory biomarkers that could explain the causal relationship between type of anesthesia used and recurrence of cancer.

In conclusion, the present study revealed no significant difference in overall survival and little difference, if any, in disease-free survival between TIVA and INHA.

\section{Abbreviations}

Pancreatic cancer (PC)

Natural killer (NK)

Gastric cancer (GC)

Stabilized inverse probability of treatment weighting (SIPTW)

Total intravenous anesthesia (TIVA)

Inhalational anesthesia (INHA)

American Society of Anesthesiologists (ASA)

American Joint Committee on Cancer (AJCC)

Disease-free survival (DFS)

Overall survival (OS)

\section{Declarations}

\section{Ethics approval and consent to participate}

This study was performed in accordance with the ethical principles of the Declaration of Helsinki. The study procedures were approved by the Ethics Committee of The Harbin Medical University Cancer Hospital. The study was registered with the Chinese Clinical Trials Registry on 03 February, 2021(URL:http://www.chictr.org.cn , Registry number: ChiCTR2200056279). This is a retrospective study and individual informed consent to participate for this retrospective analysis with routine clinical data was waived by the Ethics Committee of The Harbin Medical University Cancer Hospital.

\section{Consent for publication}




\section{Availability of data and material}

All data generated or analyzed during this study are included in this published article.

\section{Competing Interests}

The authors declare that they have no conflict of interest.

\section{Funding}

This study was supported by Postdoctoral Office of Heilongjiang Province (Hei Long Jiang Postdoctoral Foundation Grant\#LBH-Z17181).

\section{Author contributions}

Y.W. and L.W. had contributions to study conception, design, and drafting the article. J.R., J.W., Y.Y. and J.C. had contributions to acquisition and interpretation of data. Y.M., and M.W. had responsibility for the revision of important intellectual content and final approval of the version to be published.

\section{Acknowledgements}

Not applicable.

\section{References}

1. Siegel RL, Miller KD, Fuchs HE, Jemal A. Cancer Statistics, 2021. CA Cancer J Clin. 2021. 71(1): 7-33.

2. Ren C, Chen H, Han C, Fu D, Wang D, Shen M. High expression of miR-16 and miR-451 predicating better prognosis in patients with gastric cancer. J Cancer Res Clin Oncol. 2016. 142(12): 2489-2496.

3. Mohme M, Riethdorf S, Pantel K. Circulating and disseminated tumour cells - mechanisms of immune surveillance and escape. Nat Rev Clin Oncol. 2017. 14(3): 155-167.

4. Kim R. Anesthetic technique for cancer surgery: Harm or benefit for cancer recurrence. Eur J Surg Oncol. 2018. 44(5): 557-558.

5. Wang $L$, Liang S, Chen $H, X u Y$, Wang Y. The effects of epidural anaesthesia and analgesia on $T$ lymphocytes differentiation markers and cytokines in patients after gastric cancer resection. BMC Anesthesiol. 2019. 19(1): 102. 
6. Yoo S, Lee HB, Han W, et al. Total Intravenous Anesthesia versus Inhalation Anesthesia for Breast Cancer Surgery: A Retrospective Cohort Study. Anesthesiology. 2019. 130(1): 31-40.

7. Crone V, Hasselager RP, Fransgaard T, Gögenur I. Anaesthetic technique and outcomes after colorectal cancer surgery. Dan Med J. 2020. 67(4).

8. Kim R. Effects of surgery and anesthetic choice on immunosuppression and cancer recurrence. J Transl Med. 2018. 16(1): 8.

9. Herling SF, Dreijer B, Wrist Lam G, Thomsen T, Møller AM. Total intravenous anaesthesia versus inhalational anaesthesia for adults undergoing transabdominal robotic assisted laparoscopic surgery. Cochrane Database Syst Rev. 2017. 4(4): CD011387.

10. Ren XF, Li WZ, Meng FY, Lin CF. Differential effects of propofol and isoflurane on the activation of T-helper cells in lung cancer patients. Anaesthesia. 2010. 65(5): 478-82.

11. Zheng $X$, Wang $Y$, Dong $L$, et al. Effects of propofol-based total intravenous anesthesia on gastric cancer: a retrospective study. Onco Targets Ther. 2018. 11: 1141-1148.

12. Zhang $H$, Yang L, Zhu X, et al. Association between intraoperative intravenous lidocaine infusion and survival in patients undergoing pancreatectomy for pancreatic cancer: a retrospective study. Br J Anaesth. 2020. 125(2): 141-148.

13. Xu S, Ross C, Raebel MA, Shetterly S, Blanchette C, Smith D. Use of stabilized inverse propensity scores as weights to directly estimate relative risk and its confidence intervals. Value Health. 2010. 13(2): 273-7.

14. Lai HC, Lee MS, Liu YT, et al. Propofol-based intravenous anesthesia is associated with better survival than desflurane anesthesia in pancreatic cancer surgery. PLoS One. 2020. 15(5): e0233598.

15. Wu ZF, Lee MS, Wong CS, et al. Propofol-based Total Intravenous Anesthesia Is Associated with Better Survival Than Desflurane Anesthesia in Colon Cancer Surgery. Anesthesiology. 2018. 129(5): 932-941.

16. Makito K, Matsui H, Fushimi K, Yasunaga H. Volatile versus Total Intravenous Anesthesia for Cancer Prognosis in Patients Having Digestive Cancer Surgery. Anesthesiology. 2020. 133(4): 764-773.

17. Oh TK, Kim HH, Jeon YT. Retrospective analysis of 1-year mortality after gastric cancer surgery: Total intravenous anesthesia versus volatile anesthesia. Acta Anaesthesiol Scand. 2019. 63(9): 1169-1177.

18. Lim JA, Oh CS, Yoon TG, et al. The effect of propofol and sevoflurane on cancer cell, natural killer cell, and cytotoxic $\mathrm{T}$ lymphocyte function in patients undergoing breast cancer surgery: an in vitro analysis. BMC Cancer. 2018. 18(1): 159.

19. Buckley A, McQuaid S, Johnson P, Buggy DJ. Effect of anaesthetic technique on the natural killer cell antitumour activity of serum from women undergoing breast cancer surgery: a pilot study. Br J Anaesth. 2014. 113 Suppl 1: i56-62.

20. Stollings LM, Jia LJ, Tang P, Dou H, Lu B, Xu Y. Immune Modulation by Volatile Anesthetics. Anesthesiology. 2016. 125(2): 399-411.

21. Huang H, Benzonana LL, Zhao H, et al. Prostate cancer cell malignancy via modulation of HIF-1a pathway with isoflurane and propofol alone and in combination. Br J Cancer. 2014. 111(7): 1338-49.

22. Ai L, Wang H. Effects of propofol and sevoflurane on tumor killing activity of peripheral blood natural killer cells in patients with gastric cancer. J Int Med Res. 2020. 48(3): 300060520904861. 
23. Liu J, Yang L. Effects of propofol and sevoflurane on blood glucose, hemodynamics, and inflammatory factors of patients with type 2 diabetes mellitus and gastric cancer. Oncol Lett. 2020. 19(2): 1187-1194.

24. Oh CS, Lee J, Yoon TG, et al. Effect of Equipotent Doses of Propofol versus Sevoflurane Anesthesia on Regulatory T Cells after Breast Cancer Surgery. Anesthesiology. 2018. 129(5): 921-931.

25. Sargent DJ, Wieand HS, Haller DG, et al. Disease-free survival versus overall survival as a primary end point for adjuvant colon cancer studies: individual patient data from 20,898 patients on 18 randomized trials. J Clin Oncol. 2005. 23(34): 8664-70.

26. Petrelli F, Tomasello G, Ghidini M, Lonati V, Passalacqua R, Barni S. Disease-free survival is not a surrogate endpoint for overall survival in adjuvant trials of pancreatic cancer: asystematic review of randomized trials. $\mathrm{Hpb}$ the Official Journal of the International Hepato Pancreato Biliary Association. 2017: e170278.

27. Call TR, Pace NL, Thorup DB, et al. Factors associated with improved survival after resection of pancreatic adenocarcinoma: a multivariable model. Anesthesiology. 2015. 122(2): 317-24.

28. Teloken PE, Ransom D, Faragher I, Jones I, Gibbs P, Platell C. Recurrence in patients with stage I colorectal cancer. ANZ J Surg. 2016. 86(1-2): 49-53.

29. Hsu FK, Chang WK, Lin KJ, Liu CY, Fang WL, Chang KY. The Associations between Perioperative Blood Transfusion and Long-Term Outcomes after Stomach Cancer Surgery. Cancers (Basel). 2021. 13(21).

30. Hanna DN, Gamboa AC, Balch GC, et al. Perioperative Blood Transfusions Are Associated With Worse Overall Survival But Not Disease-Free Survival After Curative Rectal Cancer Resection: A Propensity ScoreMatched Analysis. Dis Colon Rectum. 2021. 64(8): 946-954.

31. Ye L, Livingston EH, Myers B, Hines OJ. The Effect of Perioperative Blood Transfusion on Long-Term Survival Outcomes After Surgery for Pancreatic Ductal Adenocarcinoma: A Systematic Review. Pancreas. 2021. 50(5): 648-656.

32. Kakar S, Pawlik TM, Allen PJ, et al Exocrine Pancreas. Pancreatic adenocarcinoma. In: Amin MB, ed. AJCC Cancer Staging Manual. 8th ed. New York, NY: Springer-Verlag; 2016.

\section{Figures}




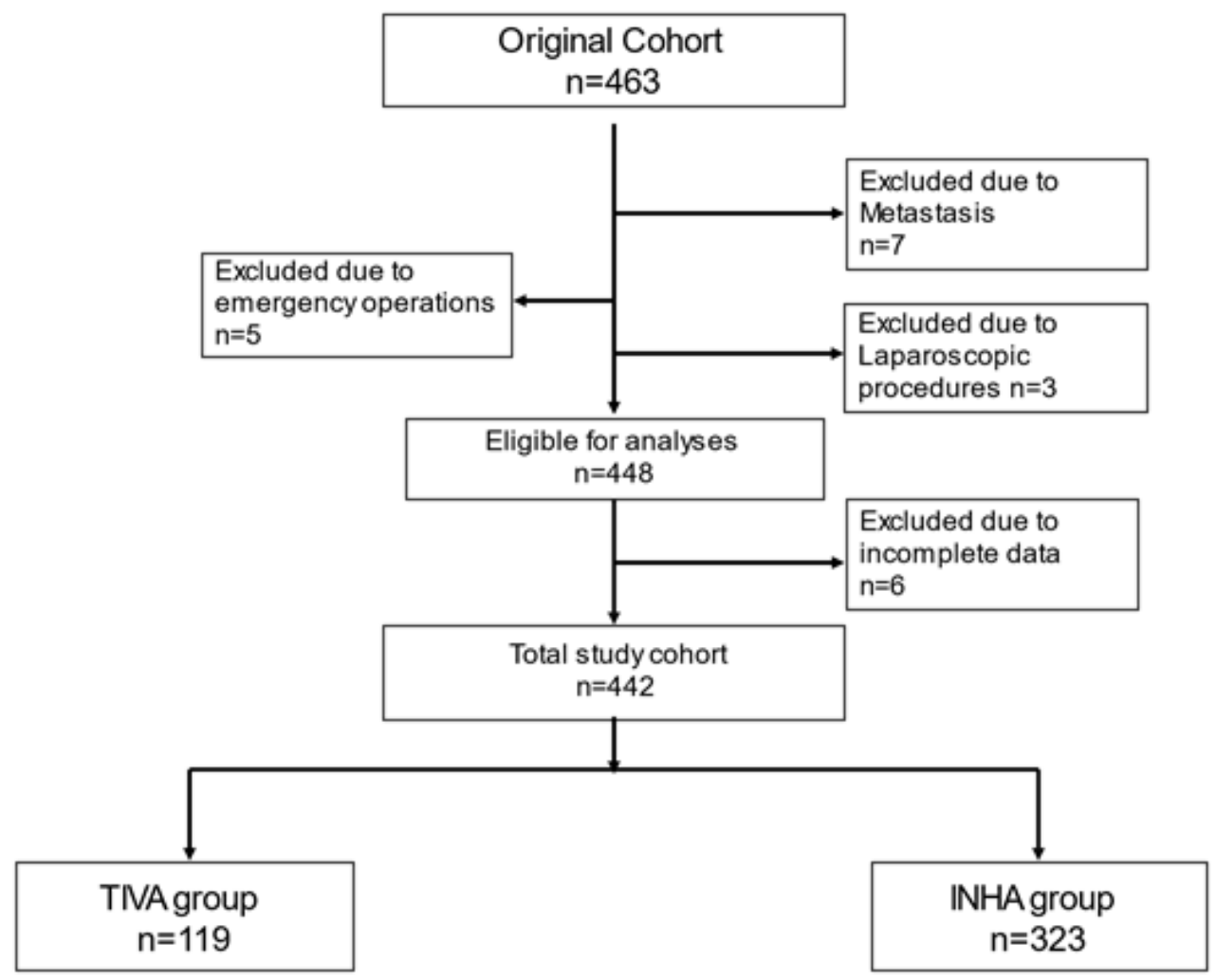

\section{Figure 1}

Patient identification and exclusion.

Abbreviations: INHA, inhalational anesthesia; TIVA, total intravenous anesthesia.

a

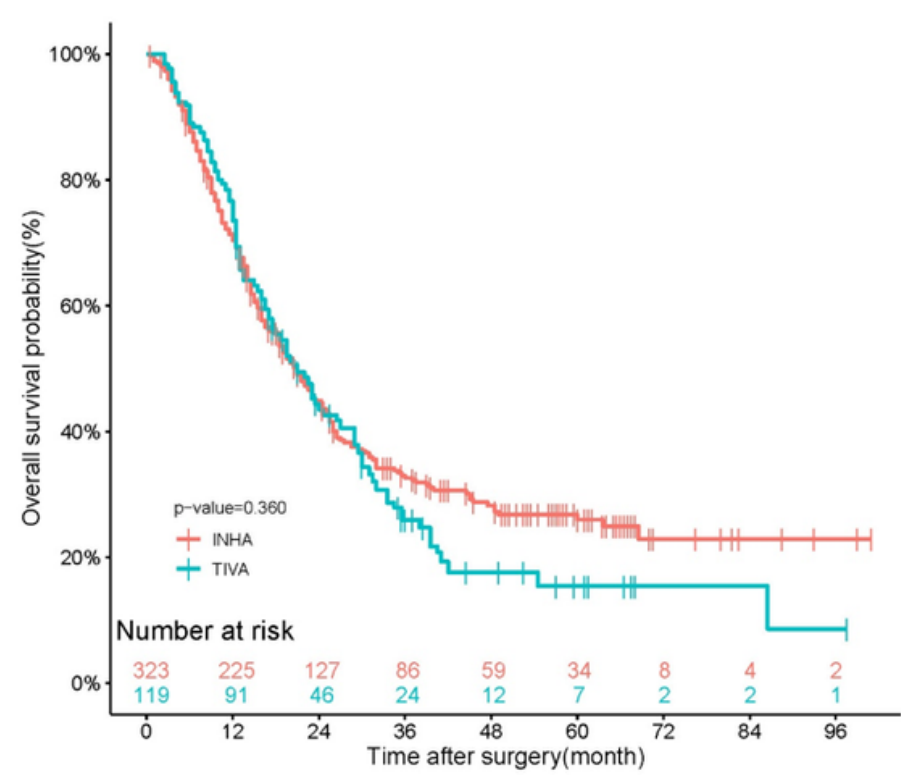

b

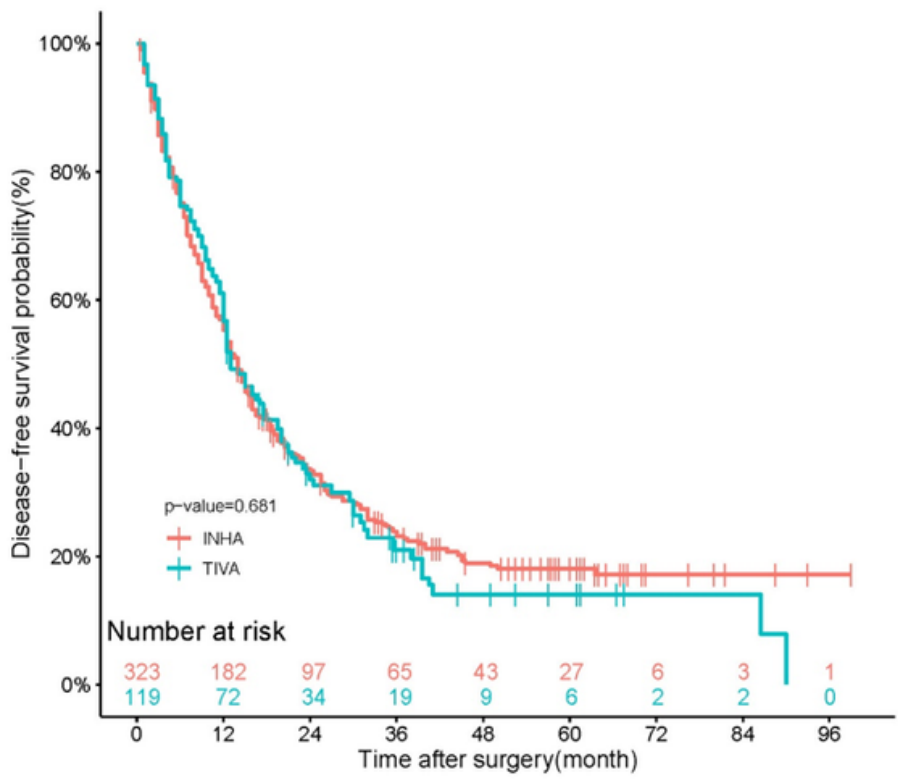

Figure 2 
Kaplan-Meier survival curve for overall survival and disease-free survival after SIPTW; SIPTW, stabilized inverse probability of treatment weighting.

a

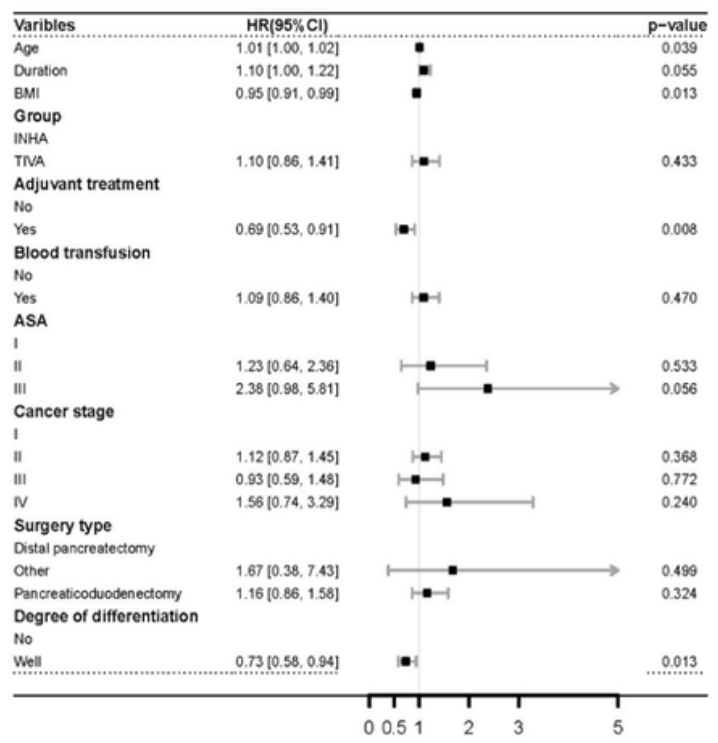

b

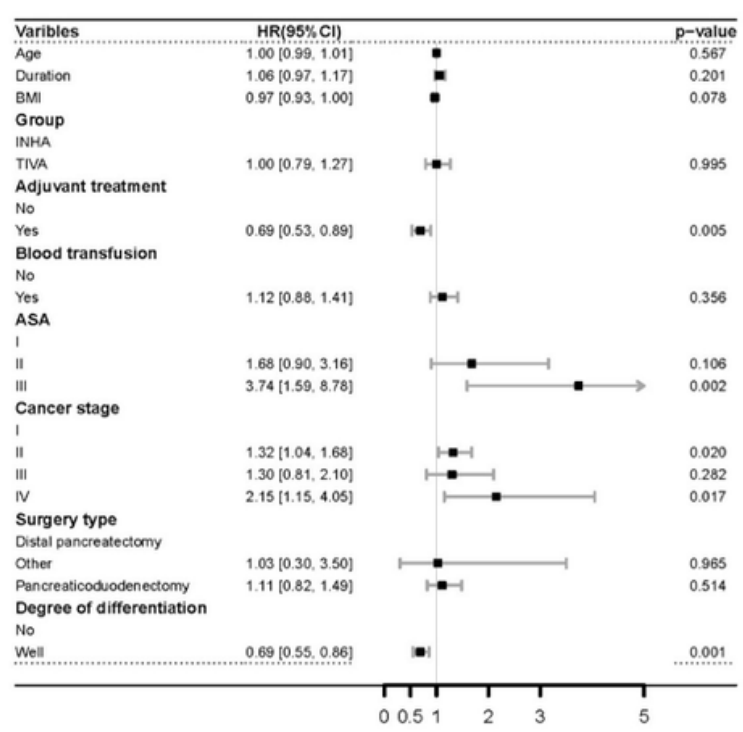

Figure 3

(a)Forest plot for multivariable cox proportional of overall survival after SIPTW (b) Forest plot for multivariable cox proportional of disease-free survival after SIPTW.ASA, American Society of Anesthesiologists $₫$ SIPTW, stabilized inverse probability of treatment weighting 